
internationales

vol. $21-n^{\circ} 3 \mid 2005$

Familles, destins personnels et appartenances collectives en migration

\title{
El vecino no deseado
}

Situación residencial de los inmigrantes africanos en Almería (España)

Juan Carlos Checa Olmos y Ángeles Arjona Garrido

\section{(2) OpenEdition}

Edición electrónica

URL: https://journals.openedition.org/remi/2529

DOI: 10.4000/remi.2529

ISSN: $1777-5418$

Editor

Université de Poitiers

Edición impresa

Fecha de publicación: 1 diciembre 2005

Paginación: 179-207

ISBN: 2-911627-45-8

ISSN: 0765-0752

Referencia electrónica

Juan Carlos Checa Olmos y Ángeles Arjona Garrido, «El vecino no deseado», Revue européenne des

migrations internationales [En línea], vol. 21 - $\mathrm{n}^{\circ} 3$ | 2005, Publicado el 01 diciembre 2008, consultado el

16 abril 2022. URL: http://journals.openedition.org/remi/2529 ; DOI: https://doi.org/10.4000/remi.2529

Este documento fue generado automáticamente el 16 abril 2022.

(c) Université de Poitiers 


\title{
El vecino no deseado
}

\author{
Situación residencial de los inmigrantes africanos en Almería (España) \\ Juan Carlos Checa Olmos y Ángeles Arjona Garrido
}

1 La segregación residencial y las deficientes condiciones de habitabilidad han sido una constante que recorre parte de la historia humana. Sin embargo, fue a lo largo del siglo pasado cuando el proceso de segregación se acentuó de manera notable, a partir de que la justificación no sólo se centraba en la clase social sino también en la edad, estado civil, religión, etnia y color de la piel, apareciendo casos extremos como el apartheid en Sudáfrica, los judíos en Europa o la segregación actual que viven los negros y algunos colectivos de hispanos en las grandes ciudades norteamericanas (véanse, entre otros, Massey y Denton, 1993; White, 1987; Bureau of Census, 2000).

2 En la actual era de las migraciones (Castles y Miller, 1998) y del mundo globalizado, en los contextos de recepción de inmigrantes seguimos asistiendo a una segregación basada en criterios étnicos y raciales. Las migraciones están creando una metamorfosis en la diversidad sociodemográfica, étnica y cultural (Santos, 1996), a la vez que generan una circunscripción vital a ámbitos espaciales determinados (Wacquant, 2001; Quilliam, 2002). Por tanto, la respuesta de las ciudades al crisol multiétnico es la exclusión y segregación espacial. Proceso que no se crea de forma «natural» tal y como lo entendieron durante décadas los autores clásicos de Chicago - Park, Burgess, Zorbaugh, etc. -, sino más bien hay que buscar la respuesta en la humanidad y los valores de la sociedad donde se insertan.

Mapa 1. Principales comarcas de agricultura intensiva y de concentración de inmigrantes (Almería).

\section{Fuente: Elaboración propia.}

3 Almería, tierra expulsora de mano de obra hacia el extranjero y otras zonas industriales de España es, en la actualidad, una provincia receptora de inmigrantes. El desarrollo de la agricultura en invernadero, a partir de los años sesenta, ha provocado la necesidad de incorporar gran cantidad de mano de obra, cubierta, en un principio, por los familiares del titular de la explotación y desde finales de los ochenta por una nueva inmigración ${ }^{1}$ procedente, fundamentalmente, de África (Checa, 1995; Roquero, 1996) y, 
más reciente, de Latinoamérica y Europa del este. Este flujo migratorio, que dura hasta nuestros días, hace que la provincia de Almería cuente con la segunda tasa de inmigración - regularizada - más alta de España y, posiblemente, la primera sumando la migración irregular. Ahora bien, la incorporación al mercado residencial de los inmigrantes no se ha producido de manera equilibrada, ni satisfactoria, ya que sufren una importante segregación y sus viviendas no cumplen con los requisitos mínimos para vivir.

\section{Datos y metodos}

El objetivo de este trabajo es doble: por un lado, cuantificar el grado de segregación de los inmigrantes y, por otro, evaluar las condiciones de dignidad de sus viviendas.

Los datos se obtienen a partir de dos fuentes diferentes. En primer lugar, los padrones municipales de los municipios y ciudades de la provincia de Almería con mayor tasa de inmigración africana - El Ejido, La Mojonera, Roquetas de Mar, Vícar (en comarca de Campo de Dalías) y Níjar (en comarca de Campos de Níjar) -. En segundo lugar, se administró un cuestionario a una muestra de la población inmigrada asentada en los lugares anteriores, el universo, el número de viviendas registradas en las fuentes oficiales es 4495 . El muestreo fue realizado por cuotas con afijación proporcional atendiendo a la nacionalidad de los ocupantes y la ubicación de las viviendas diseminado o centro urbano- con un nivel de confianza del 95,5\% y con un error muestral de $\pm 2,3$. La búsqueda de información nos llevó a muchos emplazamientos no registrados como vivienda en los diferentes padrones municipales: almacenes, chabolas, casas semiderruidas, etc. Por ello, se recurrió, por un lado, a fotos aéreas cedidas por el Ministerio de Defensa y la Universidad de Almería y, por otro, a informantes clave que demostraban la ocupación de muchos entornos no contabilizados en las fuentes oficiales. De este modo, el universo se hizo mayor y el número de alojamientos ${ }^{2}$ visitados fue de 1 648, incrementando la muestra en 81 alojamientos.

6 Para el cálculo de la segregación residencial hemos aplicado los instrumentos de medida más utilizados en la literatura internacional - Índice de Disimilitud, Aislamiento y Absoluta Centralización - (Massey, 1988), tomando como unidad espacial de análisis las manzanas. Su delimitación en los núcleos es propia y se realiza atendiendo por un lado, a sus límites geográficos y físicos y, por otro, a la fecha de construcción de los edificios. Las medidas del índice de centralización, y más concretamente en las residencias en disperso, se realiza a partir de áreas con un kilómetro cuadrado. La distancia que se toma como referencia para su medida son los edificios correspondientes a los distintos ayuntamientos o, en su defecto, delegaciones de éstos ${ }^{3}$.

7 Para la evaluación de las características y condiciones de sus viviendas partimos del concepto de vivienda digna, entendiéndola como aquella que posibilita el desarrollo normal y adecuado de sus habitantes o moradores que, a su vez, cumple con una serie de requisitos básicos articulados en los siguientes derechos (Cortés, Fernández y Plaza, 2003; Cortés, 2004).

- En primer lugar, estabilidad, que define la posibilidad de establecer un proyecto de disfrute y uso a largo plazo. Las variables principales que la determinan son régimen de tenencia, 
existencia de contratos y movilidad residencial. Esta última variable nos va a informar sobre el número de alojamientos habitados desde su llegada a la provincia de Almería.

- En segundo lugar, la adecuación, donde se establecen y definen las condiciones de la vivienda y las necesidades de sus habitantes, no sólo en el ámbito privado sino también con el entorno más próximo. De tal forma que existe inadecuación cuando las características de la vivienda impiden un uso normalizado y autónomo. Las variables que la miden son el acceso a la vivienda, características y equipamientos del entorno, el hacinamiento, la separación de los habitáculos o la percepción subjetiva que tienen los propios moradores, a través de la satisfacción que les genera el uso y disfrute de la vivienda.

- En tercer lugar, la habitabilidad, donde se hace referencia a los niveles mínimos de construcción ${ }^{4}$, servicios, infraestructuras y equipamientos de las residencias. Los elementos básicos analizados para este caso son el embaldosado, cubierta, paredes, ventilación, humedad, electricidad, agua caliente, calefacción, cocina independiente y baño integrado en la casa.

- En cuarto lugar, la accesibilidad, vinculada a una ocupación y disfrute que no suponga un gasto excesivo que afecte al desarrollo normal de otras necesidades básicas, como son la alimentación o el vestido. Por ello, la accesibilidad permite la posibilidad de uso de una vivienda en condiciones de estabilidad y seguridad económica. De este modo, tendremos en cuenta por un lado, el gasto realizado en materia de vivienda - alquiler o préstamos -, desalojos por impagos o cortes de suministros de luz y agua $\mathrm{y}$, por otro, la capacidad de endeudamiento de los moradores - tomando como referencia una inversión en vivienda del $35 \%$ de los ingresos.

8 Al mismo tiempo se ha realizado trabajo de campo con las franquicias inmobiliarias más importantes y las diferentes entidades financieras que actúan en la zona.

9 En suma, la hipótesis de partida mantiene que los inmigrantes africanos asentados en Almería, en su mayoría, están segregados y no ocupan una vivienda digna. Este hecho se explica, fundamentalmente, por el rechazo y discriminación hacia los inmigrados, contexto de recepción, y por la reducida capacidad de endeudamiento de estos demandantes, estatus económico.

\section{El habitat de los inmigrantes en almeria. un entorno dual ${ }^{5}$ entre el gueto y la ciudad dispersa}

La ubicación de los alojamientos de los inmigrantes en Almería presenta una doble tipología; por un lado están los núcleos urbanos y, por otro, los diseminados, aunque ambas formas de asentamiento comparten las características de segregación e indignidad residencial.

11 Cuando hablamos de diseminados hacemos referencia a habitáculos residenciales ubicados fuera de los núcleos urbanos - ciudad dispersa -, en suelo no urbanizable. Éste tipo de construcción residencial ha sido una constante no sólo en Almería sino en gran parte de Andalucía ${ }^{6}$ (López, 2002). Pero en la actualidad se ha consolidado como residencia permanente o temporal de los inmigrantes en muchas de las ciudades o

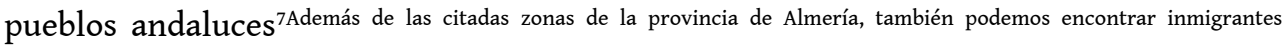
viviendo en diseminados en otros municipios del resto de provincias andaluzas. Así, por ejemplo, existen inmigrados en cortijos de Torredelcampo, Alcaudete, Villacarrillo, Martos, Úbeda, etc. en la provincia de Jaén; Zafarraya en Granada y en Huelva, entre otros, Lepe y Cartaya. 


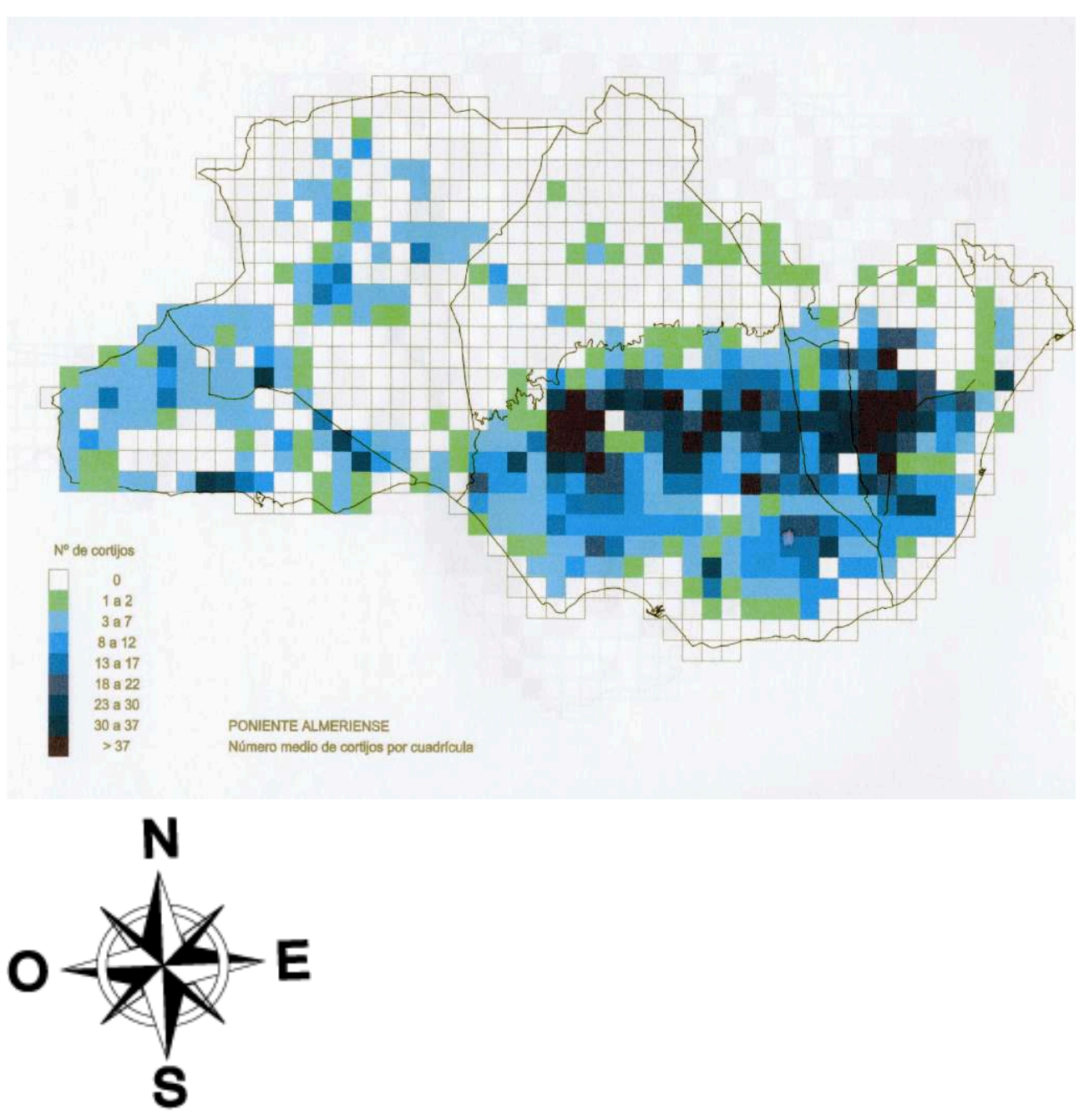

\section{La vivienda imposible}

Las condiciones y características de las viviendas que las definen como indignas se han convertido, junto a la segregación espacial, en otro hándicap para la plena integración e incorporación equilibrada de los inmigrantes africanos a la sociedad almeriense. Tomando como referencia los conceptos de residencia ${ }^{25}$ (Kemeny, 1992) y vivienda digna vamos a analizar, por separado, el grado de cumplimiento de los diferentes requistos básicos que tienen las viviendas/alojamientos de los inmigrados africanos. Aunque, en cualquier caso, la definición de vivienda digna es temporal y coyuntural e incluso depende del contexto que se analice.

\section{Estabilidad}

El alquiler es la forma más habitual de acceso a la vivienda por parte de los inmigrados africanos (55\%), seguido de la cesión (25\%) y ocupación (11\%), finalmente el $9 \%$ son propietarios. La cesión y ocupación ( $36 \%$ ) son formas que generan gran inestabilidad a los moradores de las residencias: la primera está estrechamente vinculada al 
empleador, esto es, el propietario de la vivienda la cede a los inmigrantes que se emplean, principalmente, en sus explotaciones agrícolas. Aunque esto no quiere decir que todos los ocupantes sean sus trabajadores, algunos se emplean en otras explotaciones y otros actúan como ejército de mano de obra de reserva. Además, sólo en el $35 \%$ de los casos existe un contrato que los vincula a las campañas agrícolas.

La ocupación, por su lado, es la máxima expresión de la inestabilidad por varias razones: en primer lugar, por ser lugares inhóspitos que, en la medida de sus posibilidades, se abandonarán lo más rápido posible. En segundo lugar, depende del deseo de desalojo del sitio ocupado por parte de sus propietarios ${ }^{26}$. Y, por supuesto, en ningún caso existe contrato.

La propiedad y el alquiler son formas de incorporación residencial más estables, aunque para el caso del alquiler, en ciertas ocasiones, no existe ningún contrato que regule la estancia. Pero, como ocurre con la cesión, la duración de éstos no es elevada, sólo el $3 \%$ dura tres años, dos años el $7 \%$ y un año el $15 \%$, el resto dura menos de un año, fundamentalmente entre seis o nueve meses.

Tabla 4. Acceso y título de la vivienda de los inmigrados africanos en Almería

\begin{tabular}{|l|c|l|c|}
\hline \multicolumn{2}{|c|}{ Acceso a la vivienda } & \multicolumn{2}{c|}{ Título de acceso } \\
\hline & $\%$ & & $\%$ \\
\hline Propiedad & 9 & Escritura pública & 9 \\
\hline Cesión & 25 & Contrato verbal & 23 \\
\hline Alquiler & 55 & Contrato escrito & 19 \\
\hline Ocupación & 11 & Ninguno & 48 \\
\hline Total & 100 & Otros & 1 \\
\hline & & Total & 100 \\
\hline
\end{tabular}

FUENTE: ELABORACIÓN PROPIA.

En consecuencia, la inestabilidad residencial que presentan los inmigrados es muy alta. Cuando analizamos el número de residencias habitadas desde su llegada a la provincia de Almería observamos que son numerosas. El $70 \%$ de los inmigrados declaran haber ocupado más de un alojamiento, dos el $25,5 \%$, tres el $18,9 \%$, cuatro el $15,6 \%$, cinco el $6,9 \%$ y el $3,1 \%$ seis o más. Ahora bien, en un $85 \%$ de los casos la movilidad no equivale a mejora residencial, ésta se produce de manera limitada con movimientos entre los distintos cortijos o las diferentes zonas o edificios degradados de los centros urbanos. Sólo en el $15 \%$ de las ocasiones los inmigrados africanos consiguen una movilidad residencial hacia un sitio mejor, o lo que es igual, alojamientos con mayores prestaciones - ascensor, agua caliente, habitabilidad óptima, etc. - o zonas con reconocimiento social, donde existen equipamientos e infraestructuras importantes: centros de salud y educación, lugares de ocio, tiendas, bancos, etc. Circunstancia que se produce, fundamentalmente, en el municipio de Roquetas de Mar con familias totalmente reagrupadas y con aportaciones económicas de varios miembros al núcleo familiar. En los demás municipios esta realidad se reduce, aún produciéndose las mismas condiciones, entrando en juego la política del contexto de recepción.

En consecuencia, la inestabilidad residencial afecta al $77 \%$ de los inmigrados ocupados, cedidos y alquiler de menos de un año -. Esta situación genera una gran incertidumbre y ansidedad a los moradores. 


\section{Adecuación} Almería, como vimos, es mayoritariamente en disperso, frente a la ubicación en los espacios urbanos. No obstante, en ambos casos la tipología de viviendas habitadas por los inmigrados es múltiple. De este modo, dentro de las ciudades podemos encontrar como alojamientos donde habitan inmigrados: pisos, casas independientes, cocheras, locales comerciales, huecos de escalera, terrazas de edificios, etc. $Y$ en disperso, como señalamos anteriormente, cortijos, almacenes de aperos y productos fitosanitarios, chabolas, coches abandonados, etc.

El hábitat en disperso es uno de los elementos de máxima expresión de exclusión y aislamiento residencial que, en la mayoría de los casos, deriva en una residencia no adecuada. Checa (2003) y Callejón y Casado (2004) muestran las escasas relaciones interétnicas que se producen entre los diferentes colectivos en Almería, debido, entre otros aspectos, a la ocupación de espacios diferentes, o lo que es igual, los lugares de copresencia son reducidos más allá del ámbito laboral. Por tanto, el hábitat se configura como el indicador fundamental a la hora de explicar el proceso de integración o segregación de los inmigrados ya que delimita tanto las relaciones sociales, como las laborales. Los inmigrantes deben recorrer largos trayectos entre caminos sin asfaltar, ni iluminar para llegar a los diferentes núcleos urbanos donde se sitúan la totalidad de espacios verdes, ocio, etc. de las ciudades. De igual modo, la residencia en disperso obstaculiza enormemente la incorporación laboral a otro sector que no sea la agricultura. medir su adecuación, puesto que toda vivienda debe tener un acceso que garantice la seguridad de sus habitantes. Por tanto, aceras, iluminación y señalización se convierten en elementos imprescindibles.

21 Para el primer caso, el $45 \%$ de las viviendas tienen un acceso adecuado

- rodado y peatonal o exclusivamente peatonal -, el resto tiene un acceso inadecuado (carreteras sin arcenes o aceras para viandantes) o inexistente (erial), sobre todo, en los diseminados. Por su lado, la iluminación y señalización en los diseminados es nula: sólo en los cortijos ubicados junto a los núcleos urbanos o situados en las carreteras principales tienen iluminación externa. Esta ausencia ha ocasionado el atropello de inmigrantes que transitaban por esos parajes. Del mismo modo, en los diseminados no existe un alcantarillado público, ni una recogida de basura diaria, favoreciendo los malos olores y la proliferación de ratas. Y, por supuesto, en ningún caso existe junto a los diseminados centros de salud, de educación o lugares de expansión como son parques y jardines. Esta situación ha favorecido la aparición entre los invernaderos de empresarios étnicos; es decir, inmigrados que ofrecen servicios de alimentación, ocio, teléfono, etc. a sus coétnicos (véase Arjona, 2004).

Otro de los factores que definen la adecuación de una vivienda es el hacinamiento ${ }^{27}$. Tal y como señalaba Castles y Kosack (1973) los motivos que lo generan son diversos y de origen diferente. Nosotros aquí resaltamos los más representativos e importantes para el caso almeriense: en primer lugar, los inmigrados se instalan en sitios donde comienza a existir una recarga poblacional y donde encontrar una vivienda accesible se convierte en un problema para todos los demandantes, inmigrantes y españoles. Esto 
les obliga a explotar al máximo el espacio libre que encuentran. En segundo lugar, los ingresos que se obtienen con las peonadas agrícolas son insuficientes para poder pagar de manera individual o familiar el coste de una vivienda alquilada o comprada. Por tanto, para abaratar dicho coste se reparten los gastos con otras personas ${ }^{28}$. En tercer lugar, el desconocimiento del medio y la falta de información llevan a los inmigrados, a través de las redes de apoyo, a vivir juntos. Casi todos traen una dirección en destino donde ir y allí permanecerán hasta que encuentren un trabajo u obtengan información sobre algún otro sitio. En último lugar, la permisividad de los arrendatarios. El cobro por número de moradores es más beneficioso para el arrendador, puesto que sus ingresos son mayores cuanto más inmigrados se alojen (véase Checa y Arjona, 2001; Martínez, 1999). Por tanto, el hacinamiento que en un principio parece ser sólo una estrategia de reducción de gastos del inmigrado se convierte en un negocio de rentabilidad absoluta para el propietario, ya que la vivienda alquilada a inmigrados es una mercancía en cuya conservación, reparación y adecuación no abundan las inversiones realizadas, pero sí son elevados los ingresos obtenidos.

Ahora bien, medir el hacinamiento en una residencia conlleva analizar el número de personas que viven y el espacio que disponen, pero su definición depende del momento histórico que tomemos de referencia y de los estándares definidos para la ocasión, aunque en la elaboración de éstos no existe una concepción unánime. Nosotros, tomando como referencia los estándares ofrecidos por la OCDE, el Banco Mundial y la literatura internacional más relevante, utilizamos los siguientes:

- que exista un máximo de 1,25 personas por dormitorio;

- segundo, que cada sujeto disponga de un espacio - contando los lugares comunes - de 20-25 metros;

- que exista una separación entre los dormitorios de los hijos y los padres;

- que no convivan varios núcleos familiares.

24 En cuanto al primer criterio, en Almería, los inmigrados africanos presentan de media 2,4 personas por habitación, que se eleva a 3 en los diseminados. De tal forma que sólo el 7,3\% de los africanos cumple con los estándares de 1,25 personas por habitación, sobre todo familias marroquíes que han sido reagrupadas total o parcialmente. Para el segundo caso, el $85 \%$ de los inmigrados dispone de menos de quince metros cuadrados $^{29}$. Sólo el $5 \%$ dispone de veinte metros cuadrados. El tercer y cuarto criterio de hacinamiento son los que menos presencia tienen entre los africanos: el $15 \%$ de las familias con hijos, mayores de 6 años, duermen con los padres y, en ningún momento, hermanos de distinto sexo mayores de 16 años duermen juntos. De forma similar, sólo en el $12 \%$ de los casos conviven varios núcleos familiares, realidad que es más común entre los europeos del este y, más concretamente, los rumanos. En cualquier caso, el hacinamiento se produce tanto en residencias en régimen de alquiler, como de cesión, ocupación e incluso de propiedad.

En último lugar, la adecuación también viene definida por la percepción que tienen los propios habitantes. Para nuestro caso sólo el $8 \%$ está totalmente satisfecho con las oportunidades que le ofrece su vivienda y entorno. Sin embargo, el $76 \%$ de los inmigrados africanos declaran insatisfacción.

Las causas esgrimidas de la insatisfacción son: en primer lugar, las condiciones de habitabiliad de la vivienda y las deficientes condiciones de los caminos/calles, especialmente para los habitantes de las chabolas, almacenes y cortijos. En segundo lugar, lo poco adecuadas que son para un desarrollo vital normal, sobre todo, por las 
distancias a recorrer para realizar la compra, ocio o llevar a los hijos a los centros educativos. En ningún caso existen autobuses urbanos que ofrezcan el servicio de recogida de personas entre los invernaderos. De tal forma que los desplazamientos se realizan, generalmente, a pie o en bicicleta. En tercer lugar, el elevado coste económico de la vivienda con respecto a los ingresos obtenidos, o lo que es igual, escasa capacidad de endeudamiento. Por último, el hacinamiento que padecen en los alojamientos.

Tabla 5.Grado de satisfacción con la casa

\begin{tabular}{|l|c|}
\hline & $\%$ \\
\hline Totalmente satisfecho & 8 \\
\hline Bastante satisfecho & 13 \\
\hline Poco satisfecho & 54 \\
\hline Nada satisfecho & 22 \\
\hline NS/NC & 3 \\
\hline Total & 100 \\
\hline
\end{tabular}

FUENTE: ELABORACIÓN PROPIA.

En resumen, el $93 \%$ de los inmigrados habitan en alojamientos donde se da un indicador de inadecuación, el $77 \%$ con dos y el $58 \%$ con tres.

\section{Habitabilidad}

La habitabilidad de las viviendas se convierte en el elemento, por excelencia, para la definición de una residencia digna. Los indicadores elegidos para su medición son la calidad de la construcción y la tenencia de infraestructuras básicas.

De la calidad de la construcción depende la seguridad de los ciudadanos, de tal forma que una vivienda con condiciones deficientes en la cubierta, paredes o suelo pone en grave peligro la vida de los habitantes, de forma parecida la ventilación o la humedad definen la habitabilidad residencial. Para nuestro caso, los alojamientos habitados por los inmigrados africanos, el $43 \%$ presenta alguna de esas deficiencias, el $32 \%$ dos y el $21 \%$ todas. Inhabitabilidad que se dan mayoritariamente en los diseminados, puesto que en los núcleos urbanos sólo se produce en el $11 \%$ de las viviendas. Sin embargo, según el censo de viviendas de 2001, sólo el 6 \% de las viviendas ocupadas por españoles presentan alguna de esas deficiencias.

La habitabilidad de la vivienda también viene determinada por tener agua potable y caliente, electricidad o calefacción/refrigeración: el $68 \%$ tiene agua potable, el $55 \%$ agua caliente y el $71 \%$ tiene electricidad, sin embargo, sólo el $0,3 \%$ de las viviendas tienen calefacción o aire acondicionado. Cifras que también distan bastante de las viviendas ocupadas por españoles: el $95 \%$ tiene agua potable, el $93 \%$ agua caliente y el $99 \%$ electricidad.

31 En los alojamientos que no disponen de agua potable los inmigrantes la recogen en las balsas de almacenamiento que dan riego a los invernaderos, corriendo un grave riesgo 
de insalubridad tanto por su consumo directo, como en las comidas o el aseo personal. De forma similar, en los alojamientos que no disponen de luz eléctrica se utilizan velas o linternas para su iluminación.

Asimismo, la existencia de determinadas unidades habitacionales y su separación del resto es fundamental. En el $75 \%$ de los casos existe una cocina u hornilla para cocinar, pero el $35 \%$ encuentra, normalmente, en el salón o fuera del propio alojamiento. Lo mismo ocurre con el baño, existe en el $72 \%$ de los alojamientos, pero el $38 \%$ no tiene una ubicación separada o su construcción está ubicada fuera. Hecho que se repite con los dormitorios, en el $18 \%$ de las viviendas algún dormitorio coincide con el salón.

Del compendio de todos los factores resulta que el $43 \%$ de los inmigrantes habitan en alojamientos que padecen la ausencia de alguno de los anteriores elementos fundamentales, que afecta a la inhabitabilidad de las viviendas.

\section{Accesibilidad}

El último indicador que define la dignidad de una vivienda es su accesibilidad, o lo que es igual, la inversión económica que debe hacer una persona para poder acceder a ella. Las encuestas nacionales de presupuestos y gastos familiares han puesto de manifiesto que los costes del uso y ocupación de la vivienda son muy elevados ${ }^{30}$, hecho que se agrava en el caso de los inmigrantes, aquiriendo tintes dramáticos.

Todos los inmigrados africanos que afirman vivir en régimen de propiedad en Almería han necesitado ayuda financiera de otras fuentes: $95 \%$ exclusivamente de bancos y el resto de familiares y/o amigos y bancos. En cualquier caso, el coste mensual medio de sus pagos asciende a 330,5€. Sin embargo, la media de ingresos es de 611,9 $€$, dicho de otro modo, más de la mitad de los ingresos (54\%) van destinados, exclusivamente, al pago de la vivienda. Ahora bien, normalmente, las familias con viviendas en propiedad tienen varios miembros trabajando fuera del hogar, además del sueldo del cabeza de familia hay otras aportaciones para el pago de los préstamos de la residencia, hasta el punto que el gasto familiar en vivienda asciende al $38 \%$ de su presupuesto total.

Por su lado, el coste medio de los inmigrados que residen en casas de alquiler es de $114,76 €$ al mes, lo que equivale al $18,75 \%$ de sus ingresos. Este dato, que a priori no es elevado, esconde una realidad diferente, puesto que en ningún caso el inmigrado vive sólo, comparte casa en condiciones de hacinamiento, entre otras razones, para abaratar los costes. Evidentemente, esta realidad limita otros derechos fundamentales: reagrupamiento familiar, intimidad, adecuación, etc. Por tanto, si el inmigrado tuviera que hacer frente al pago sin ayuda de los demás la inversión media ascendería al $85 \%$ de sus ingresos. 
Tabla 6. Accesibilidad residencial de los inmigrados africanos en Almería.

\begin{tabular}{|c|c|c|c|c|c|}
\hline \multicolumn{2}{|c|}{$\begin{array}{l}\text { Coste mensual del alquiler } \\
\text { (individual) }\end{array}$} & \multicolumn{2}{|c|}{$\begin{array}{c}\text { Coste mensual de } \\
\text { hipoteca } \\
\text { (total vivienda) }\end{array}$} & \multicolumn{2}{|c|}{ Ingresos mensuales } \\
\hline$€$ & $\%$ & $€$ & $\%$ & $€$ & $\%$ \\
\hline $0-30$ & 2,1 & $120,01-180$ & 3,1 & $120,01-180$ & 0,4 \\
\hline $30,01-60$ & 32,6 & $180,01-240$ & 8,1 & $180,01-240$ & 0,8 \\
\hline $60,01-90$ & 27,9 & $240,01-300$ & 21,5 & $240,01-300$ & 1,1 \\
\hline $90,01-120$ & 14,5 & $300,01-360$ & 26,0 & $300,01-360$ & 1,3 \\
\hline $120,01-150$ & 9,0 & $360,01-420$ & 18,8 & $360,01-420$ & 3,9 \\
\hline $150,01-180$ & 6,2 & $420,01-480$ & 9,2 & $420,01-480$ & 4,4 \\
\hline $180,01-210$ & 3,4 & $480,01-540$ & 4,6 & $480,01-540$ & 8,6 \\
\hline Más de 210 & 4,3 & $540,01-600$ & 4,8 & $540,01-600$ & 8,9 \\
\hline Coste medio & $114,76 €$ & $600,01-660$ & 2,7 & $600,01-660$ & 9,4 \\
\hline & & Más de 660 & 1,2 & $660,01-720$ & 7,5 \\
\hline & & Coste medio & $330,5 €$ & $720,01-780$ & 19,4 \\
\hline & & & & $780,01-840$ & 14,5 \\
\hline & & & & $840,01-900$ & 6,5 \\
\hline & & & & $900,01-960$ & 6,2 \\
\hline & & & & $960,01-1020$ & 5 \\
\hline & & & & Más de 1020 & 2,1 \\
\hline & & & & Ingresos medios & $611,97 €$ \\
\hline
\end{tabular}

FUENTE: ELABORACIÓN PROPIA

Los inmigrados que residen en régimen de cesión ${ }^{31}$, en teoría, no pagan nada por el alojamiento. Pero en casí la mitad de los casos (42\%) la realidad es diferente: los empresarios no cobran un alquiler directamente, aunque los inmigrados pagan su residencia a través de trabajos fuera de la jornada laboral - subir y bajar las bandas del invernadero, regar de madrugada, vigilar la explotación - o, en otros casos, se resta del sueldo. Concretamente para esta última forma, el convenio del campo es de $35 €$ por día, pero un escaso $25 \%$ de los empresarios paga esa cantidad, el resto paga entre 27 y $30 €$, tomando como excusa la cesión de la vivienda. Para este caso los cálculos demuestran que el ahorro en el pago salarial es superior a los ingresos que se obtendrían con el alquiler, debido a que el arrendatario deja de pagar $5 €$ por día, lo que al mes supone $150 €$, cantidad superior a la pagada como media por un alojamiento alquilado. Por ello, estamos hablando de micromercados de base local ${ }^{32}$, parcialmente desgajados de la competencia general (Bourdieu, 2003).

Los impagos de recibos de luz y agua son comunes, el $43 \%$ de los inmigrados en algún momento no han podido hacer frente a las facturas a tiempo, pagando posteriormente el recargo o el nuevo enganche. Además, el $12 \%$ de los inmigrados han sido desalojados en algún momento fruto de las políticas higienistas locales, aunque en ningún caso se ha ofrecido alguna alternativa residencial, por lo que han vuelto a construirse o reubicarse en otro sitio de iguales o peores características. En suma, el $31 \%$ de los inmigrados tienen problemas de accesibilidad en su vivienda actual, aunque el resto para conseguirla recurran al hacinamiento o la ayuda económica de uno o varios miembros familiares.

En conclusión, y derivado de la acumulación de carencias en los requisitos de adecuación, accesibilidad, estabilidad y habitabilidad, podemos mantener que el $71 \%$ de los inmigrados africanos asentados en la provincia de Almería residen en infraviviendas ${ }^{33}$. 


\section{El vecino no deseado en un mercado residencial rehabilitado}

40 Los motivos que explican la ocupación de viviendas no dignas son múltiples y de diverso calado. Según se desprende del análisis de regresión ${ }^{34}$ el primer motivo que explica las malas condiciones de habitación es la discriminación percibida: cuanto mayor es la discriminación peores condiciones de habitabilidad tienen los alojamientos. Los inmigrantes aseguran que aun teniendo capacidad económica son muchas las trabas que encuentran para ocupar una vivienda digna. En la encuesta administrada a los inmigrados el $30 \%$ de ellos afirman que estarían dispuestos hasta pagar unos $300 €$ mensuales por una casa con unas buenas condiciones.

41 De ahí, que los principales lugares ocupados son diseminados esparcidos entre invernaderos, donde es mayor su invisibilidad. Pero cuando habitan los núcleos urbanos se concentran en determinados barrios o edificios con mayor deterioro. La explicación sobre la discriminación tiene dos vectores: uno, como vimos anteriormente, la preferencia de los autóctonos como vecinos de unos colectivos frente a otros (Arjona, 1999). Dos, intereses económicos, ya que se rehabilita un mercado residencial de escasa solvencia, caracterizado por viviendas con importantes deficiencias, donde no se invierte nada y, por supuesto, ningún autóctono está dispuesto a ocupar.

El segundo elemento que define una vivienda no digna es su ubicación. De tal forma que las viviendas/alojamientos en disperso son las que peores condiciones presentan en todos los aspectos. Situación que se hace dramática, sobre todo, en Campos de Níjar, donde los inmigrados africanos están condenados a residir en los diseminados, puesto que la población autóctona tiene muchas reticencias a tenerlos como vecinos.

43 Así, por ejemplo, junto al municipio de San Isidro hay un invernadero de unos siete mil metros cuadrados en deshuso, donde se han ubicado más de cien chabolas que dan cobijo, según la época, a cuatrocientos o quinientos subsharianos, principalmente malienses y senegaleses. El invernadero se ha convertido en una pequeña ciudad sin infraestructuras, ni equipamientos. Recientemente, el Ayuntamiento de Níjar proporcionó un grifo con agua potable junto al invernadero, aunque con anterioridad el agua era recogida a más de un kilómetro, en el centro del pueblo. El $80 \%$ de estos inmigrantes llevan viviendo más de dos años en esas condiciones, con unas expectativas nulas de salir de ahí. Todos se emplean en la agricultura, excepto un inmigrante que se dedica a arreglar las bicicletas del resto y otro que calienta agua, en un fuego de leña, durante todo el día para todos aquellos que la precisen.

También podemos encontrar en dicha comarca a inmigrados alojados en antiguas cochiqueras y cuadras de animales, así como coches, furgonetas o el remolque de un camión. Por ello, podemos afirmar, sin temor a equivocarnos, que todos estos lugares han pasado a ser enclaves de superviviencia y pobreza extrema.

El tercer elemento explicativo de la ocupación de infraviviendas lo hemos llamado temporalidad. Queda definido por los años de estancia, documentación y conocimiento e información sobre el entorno, de tal forma que cuanto más tiempo se lleve residiendo, mejores son las condiciones residenciales adquiridas puesto que, por un lado, la documentación puede ser un freno superado y, por otro, el tiempo de asentamiento 
favorece un conocimiento mayor, en todos los aspectos, de la dinámica de la sociedad de llegada.

Sin embargo, este elemento tiene determinadas aristas que vuelven a remitir a la discriminación étnica, dado que los inmigrantes procedentes de África son los que mayor tiempo de estancia acumulan en la provincia de Almería, frente a los latinoamericanos y rumanos, cuya presencia masiva se produce a partir de 2000, pero ocupan mejores viviendas. De forma parecida, la irregularidad afecta más a los latinoamericanos y europeos del este, su reciente llegada a España les ha impedido regularizar su situación en las diferentes regularizaciones extraordinarias, lo que si han podido hacer de maner «más fácil» los subsharianos que han demostrado una estancia más larga en nuestro país.

El cuarto elemento es la capacidad de endeudamiento de los inmigrados africanos. Cuanto mayores son los ingresos, mejores son las condiciones residenciales. Como veíamos anteriormente gran parte de la población africana tiene importantes problemas de accesibilidad a la vivienda, invirtiendo más del $35 \%$ de sus ingresos. Ahora bien, significa esto que los inmigrados llegados de otros lugares ganan más dinero o están dispuestos a invertir más cantidad de dinero en la vivienda. Checa (2004) muestra que las diferencias salariales entre los diferentes grupos no son tan elevadas. Si bien es cierto que los africanos parecen condenados a insertarse laboralmente en los invernaderos, quedando su movilidad laboral más reducida y con menor salario, los otros grupos poblacionales se emplean, además de la agricultura, en su industria auxiliar, en la construcción, servicio doméstico o restauración. Pero en todos los casos se trata de empleos con grandes dosis de economía sumergida y de baja remuneración. Así, excepto en las viviendas cedidas y/o ocupadas los precios pagados son similares, aunque con diferentes condiciones de habitabilidad, adecuación y estabilidad, por tanto, otra vez, se reafirma la discriminación como el elemento fundamental que explica esta situación.

La reagrupación familiar y las preferencias residenciales se erigen como el quinto y el sexto elemento explicativo de una vivienda no óptima. La presencia de la mujer es un elemento fundamental para el acceso a una vivienda digna. La población autóctona pone menos trabas cuando la familia está completa, debido a la suposición de un mejor mantenimiento por la presencia femenina. Y, evidentemente, las preferencias residenciales no son muy explicativas de dicha situación, puesto que éstas quedan muy reducidas, no más allá del mercado residencial diseñado para ellos. 
Tabla 7. Variables que inciden en la ocupación de una vivienda no digna en Almería

\begin{tabular}{|l|c|c|}
\hline & B & Beta \\
\hline Ubicación & $-0,43$ & $-0,98$ \\
\hline Preferencias residenciales & 0,01 & 0,08 \\
\hline Reagrupamiento familiar & 0,18 & 0,15 \\
\hline Años de residencia & $-0,41$ & $-0,60$ \\
\hline $\begin{array}{l}\text { Percepción de discriminación en venta y alquiler de } \\
\text { la población autóctona y las agencias inmobiliarias }\end{array}$ & 0,54 & 1,18 \\
\hline Accesibilidad -ingresos- & $-0,28$ & $-0,35$ \\
\hline Constante & \multicolumn{2}{|c|}{15,6} \\
\hline $\mathrm{R}^{2}$ & \multicolumn{2}{|c|}{0,76} \\
\hline
\end{tabular}

Fuente: Elaboración propia

B: Coeficientes de Regresión parcial; Beta: Coeficiente de regresión estandarizado. Sig T=0.00 No se incluyen las variables independientes que no cumplen los criterios de significación y con Beta menor de 0,1 .

Las variables nominales y ordinales fueron transformadas en dummy.

\section{Conclusiones}

Las cifras mostradas a lo largo del texto reflejan que la población africana asentada en Almería sufre altas tasas de segregación y un derecho de habitar truncado, puesto que casi tres de cada cuatro inmigrados se aloja en una vivienda no digna. El principal hábitat que ocupan estos inmigrantes son los dispersos - cortijos, almacenes de aperos, antiguos cementerios, transformadores de luz, aljibes, etc. - situados entre el laberinto de caminos, veredas o eriales que componen el campo, que por su ubicación los deja invisibles. Por tanto, los inmigrantes en Almería habitan en enclaves de pobreza, que en la actualidad sólo son espacios de supervivencia. Así, se pone al descubierto una línea étnica urbana que separa a los segregados e indeseables del resto. Frontera que tiene su principal base en la discriminación deliberada desde todos los ámbitos: ciudadanos, actores políticos, entidades financieras, administraciones, etc., de ahí, que estemos ante una miseria modernizada, donde todo el mundo tiene responsabilidad pero nadie se hace responsable.

Esta línea urbana, casi dual, genera un mercado residencial segmentado donde la diversidad no es relacional, sino que está producida en base a las condiciones del contexto social en que se da. También es jerarquizante y desigualitario porque implica juicios de valor y relaciones de poder. Esto es, se produce y define al «diferente» al que no se quiere como vecino $\mathrm{y}$, además, rehabilita un mercado residencial de nula rentabilidad controlado, en mayor medida, por los empleadores y no, tanto, por las agencias inmobiliarias.

51 La reducida capacidad económica de los inmigrantes, explicada en parte por un mercado de trabajo segmentado, discontinuo y flexible con una mínima movilidad laboral, junto a la ubicación de los alojamientos, el tiempo de residencia o las preferencias residenciales de los inmigrados también explican, aunque en menor medida, la segregación y ocupación de hábitats indignos.

En resumen, sea como fuere, la situación residencial de los inmigrados africanos en la provincia de Almería es dramática y precisa de una actuación rápida y eficaz que solvente este problema, que está derivando en otros: segregación escolar, proliferación 
de enfermedades infecto-contagiosas, etc., o lo que es igual, injusticia social y ambiental.

\section{BIBLIOGRAFÍA}

ABU-LUGOD Janet (1997) The specifity of the Chicago ghetto: comment on Wacquant's, International Journal and Regional Research, $\mathrm{n} \div 21$.

ANDERSON James (2004) Whites and their neighbours: trends, explanations and neighbourhoodlevel outcomes of white residential segregation 1970-2000, The Humanities and Social Science, vol. $64, \mathrm{n}^{\mathrm{o}} 7$.

ARJONA Angeles (1999) Racismo y xenofobia en el Poniente almeriense. Universidad de Almería. Memoria de doctorado, Sin Publicar

ARJONA Angeles (2004) Inmigración y mercado de trabajo. El caso de la economía étnica en Almería, Almería, Universidad de Almería.

BANCO MUNDIAL (1992) The housing indicators program extensive survey. Preliminary results, Washington DC, World Bank.

BENEVOLO Leonardo (1980) The history of the city, London, Scholar Press.

BERRY, John (1979) The open housing question: race and housing in Chicago, 1966-1976, Cambridge, Ballinger.

BLAKELY Edwards and GAIL Mary (1997) Fortress America. Gated communities in the United States, Cambridge, Lincoln Institute of Land Policy.

BOURDIEU Pierre (2003) Las estructuras sociales de la economía, Barcelona, Anagrama.

BRUCE Roger (2001) The geography of residential and employment inequality: workplace and home place in urban space, Madison, University of Wisconsin.

CALLEJÓN David (2004) Integración y relaciones de convivencia entre los jóvenes autóctonos e inmigrados en Almería, en Actas del IV Congreso Nacional de Inmigración, Girona.

CALLEJÓN David y CASADO Aurora (2004) Inmigración y relaciones de convivencia entre los jóvenes autóctonos e inmigrados en Almería, en Actas del VIII Congreso Nacional de Sociología de España, CD-ROM.

CASTLES Stephen and KOSACK Godula (1973) Immigrants, workers and class structure in Western Europe, London, Oxford University Press.

CASTLES Stephen and MILLER Mark (1998) The age of migrations. Population movements in modern world, London, Palgrave.

CHECA Francisco (1995) Oportunidades socioeconómicas en el proceso migratorio de los inmigrantes africanos en Almería, Agricultura y Sociedad, nº 77, pp. 83-108

CHECA Francisco (2003) Inmigración y diversidad en España. Una aproximación desde el extrañamiento cultural, Convergencia, $\mathrm{n}^{\circ} 33$, pp. 139-175. 
CHECA Juan Carlos (2004) La diferenciación residencial de los inmigrados en la provincia de Almería: el caso de Roquetas de Mar, Almería, Universidad de Almería.

CHECA Juan Carlos y ARJONA Angeles (2001) El Ejido: de erial urbano a ciudad-cortijo, en Francisco Checa Dir., El Ejido: la ciudad-cortijo. Claves socioeconómicas del conflicto étnico, Barcelona, Icaria, pp. 127-170.

CORTÉS Luís (1995) La cuestión residencial. Bases para una sociología del habitar, Madrid, Fundamentos.

CORTÉS Luís (2004) Vivienda y exclusión social, en Actas del VIII Congreso Nacional de Sociología de España, CD-ROM.

CORTÉS Luís, FERNÁNDEZ Celia y PLAZA Pilar (2003) Vivienda y exclusión residencial, en M. Aguilar; M. Laparra; B. Pérez Coord., Investigaciones de base para la elaboración del plan de lucha contra la exclusión social en la Comunidad de Madrid. Madrid, Comunidad de Madrid y Universidad Pública de Navarra, pp. 285-405.

DEFENSOR DEL PUEBLO ANDALUZ (2001) El alojamiento y la vivienda de los trabajadores inmigrantes en el Poniente almeriense y Campo de Níjar, Sevilla, Junta de Andalucía.

DÍEZ Juan Dir. (1998) Actitudes hacia los inmigrantes, Madrid, IMSERSO.

DOHERTY Paul and POOLE Michael (1995) Ethnic residential segregation in Belfast, Belfast, Centre for the Study of Conflict.

DOWNEY Liam (2003) Spatial measurement, geography, and urban racial inequality, Social Forces, vol. 81, no 3, pp. 937-952.

ENGELS Friedrich (1965) La situación de la clase obrera en Inglaterra, Madrid, Akal.

FISHER Robert (1984) Let the people decide: neighborhood organizing in America, Boston, Twayne. FORO CÍVICO (2000) El Ejido tierra sin ley, Navarra, Lizarra.

FRIEDRICHS Jürgen (1998) Ethnic segregation in Cologne, Germany 1984-94, Urban Studies, ํㅜ 35, pp. $1745-1763$.

GIFFINGER Rudolph (1998) Segregation in Vienna: impacts of market barriers and rent regulations, Urban Studies, no 35, pp. 1791-1812.

GIRÓN Carlos (2002) El alojamiento y la vivienda del colectivo inmigrante en las provincias. En L. Serra Éds. Inmigración extranjera en Andalucía. II Seminario sobre la investigación de la inmigración extranjera en Andalucía, Sevilla, Junta de Andalucía, pp. 91-101.

GRANNIS Rick (2002) Discussion: segregation index and their functional inputs, Sociological Methodology, no 32, pp. 69-84.

HOGGART Keith y MENDOZA Cristóbal. (1999) African immigrant workers in spanish agriculture, Sociologia Ruralis, nํ39, pp. 538-562.

HUTTMAN Elizabeth, BLAUW Wim and SALTMAN Juliet R. Edits (1991) Urban housing segregation of minorities in western Europe and United States, London, Duke University Press.

KAIN John (1965) The effect of the ghetto on the distribution and level of nonwhite employmentin urban areas, Proceedings, Social Statistics Section of The American Statistical Association.

KAIN John (1968) Housing segregation, negro employment and metropolitan decentralization, Quarterly Journal of Economics, ํㅡㅇㅛ 82, pp. 175-197. 
KEMENY Jim (1992) Housing and social theory, London, Routledge.

KEMPER Franz-Josef (1998) Restructuring of housing and ethnic segregation: recent developments in Berlin, Urban Studies, nº 35 pp. 1765-1789.

LIEBERSON Stanley (1980) A piece of the pie. Blacks and white immigrants since 1880, Los Angeles, University of California Press.

LÓPEZ Antonio (2002) Las agrociudades andaluzas, en E. Moyano y M.Pérez Éds., La sociedad andaluza, Córdoba, IESA, pp. 285-305.

LURBE Kàtia (2004) La gestión de inmigrantes extracomunitarios y refugiados como grupos de riesgo sanitario, en Actas del IV Congreso sobre la inmigración en España: ciudadanía y participación, En CD-Rom.

MALHEIROS Jorge (2002) Ethni-cities: Residential patterns in the northern European and Mediterranean metropolises. Implications for policy design, International Journal of Population Geography, nº 8 pp. 107-134.

MARTÍN Emma, MAYNAR Ana y SANZ Gonzalo (2001) Mercados de trabajo e inmigración extracomunitaria en la Agricultura Mediterránea, Sevilla, Junta de Andalucía.

MARTÍNEZ Ubaldo (1999) Pobreza, segregación y exclusión espacial. La vivienda de los inmigrantes extranjeros en España, Barcelona, Icaria.

MARTÍNEZ Ubaldo (2001) El Ejido. Discriminación, exclusión social y racismo, Madrid, Catarata.

MARTORI Juan Carlos y HOBERG Karen (2003) La segregació residencial de la població estrangera a Catalunya, Fundació Jaume Bofill, Barcelona.

MASSEY Douglas and DENTON Nancy (1988) The dimensions of residential segregation, Social Forces, vol. 67, nº 2, pp. 281-315.

MASSEY Douglas and DENTON Nancy (1989) Hypersegregation in U.S. metropolitan areas: Black and Hispanic segregation among five dimensions, Demography, vol. 26, no 3, pp. 373-391.

MASSEY Douglas and DENTON Nancy (1993) American apartheid, Cambridge, Harvard University Press.

MEYERSON Martin and BANFIELD Edwards (1955) Politics, planning and the public interest, Glencoe, The Free Press.

MINGIONE Enzo (1996) Urban Poverty and the underclass: a reader, Cambridge, Balckwell.

MOTTURA George (1992) Forme della presenza extracomunitraia nella agricoltora italiana: resultadi di una prima exploracione, Ponencia defendida en el curso Trabajadores inmigrantes en la agricultura mediterránea, Valencia.

MUSTERD Sako and DEURLOO Rinus (2002) Unstable Immigrant Concentrations in Amsterdam: Spatial Segregation and Integration of Newcomers, Housing Studies, no 17, pp. 487-503.

PEACH Ceri (2001) The ghetto and the ethnic enclave, International Seminar of Segregation in the City, Cambridge, Lincoln Institute of Land Policy.

PETSIMERIS Petros (1998) Urban decline and the new social and ethnic divisions in the core cities of the italian industrial triangle, Urban Studies, $\mathrm{n}^{\circ}$ 35, pp. 449-465.

PORTES Alejandro y BÖRÖCZ József (1992) Inmigración contemporánea: perspectivas teóricas sobre sus determinantes y modos de acceso, Alfoz, no 91-92, pp. 20-33. 
PUGLIESE Enrico (1992) L'immigraziones in Italia: un confronto tra nord e sud, Ponencia defendida en el curso Trabajadores inmigrantes en la agricultura mediterránea, Valencia.

QUILLIAN Lincoln (2002) Why is black-white residential segregation so persistent ?: evidence on three theories from migration data, Social Science Research, vol. 31, no2, pp. 197-229.

REARDON Sean and FIREBAUG Glenn (2002) Measures of Multigroup segregation, Sociological Methodology, no 32, pp. 32-67.

ROQUERO Esperanza (1996) Asalariados africanos segregados bajo plástico, Sociología del Trabajo, no 28 , pp. 3-24.

SANTOS Milton (1996) Metamorfosis del espacio habitado, Barcelona, Oikos-tau.

SJOBERG Gideon (1960) The preindustrial city: past and present, New York, Free Press.

TURNER John (1968) Housing priorities, settlement patterns and urban development in modernizing countries, Journal of the American Institute of Planners, $n^{\circ}$ 34, pp. 53-71

TURNER John (1972) Housing as a Verb, en J. Tuner y R. Ficter Eds., Freedom to Build, New York, Macmillan.

US BUREAU OF THE CENSUS (2000) Census of Population and Housing.

VANCE James (1971) Land assignment in pre-capitalist, and post-capitalist cities, Economic geography, nº 47, pp. 101-120.

WACQUANT Loïc (1989) The Ghetto, the State and the New Capitalist Economy, Dissent, pp. 508-520.

WACQUANT Loïc (1997) Three pernicious premises in the study of the American ghetto, International Journal of Urban and Regional Research, vol. 21, no 2, pp. 456-473.

WACQUANT Loïc (2001) Parias Urbanos. Marginalidad en la ciudad a comienzos del milenio, Buenos Aires, Manantial.

WHITE Michael (1987) American neighborhoods and residential differentiation, New York, Russel Sage Foundation.

WILSON William (1978) The declining significance of race, Chicago, The University of Chicago Press.

WILSON William (1987) The truly disadvantaged. The inner city, the underclass, and public policy, Chicago, The University of Chicago Press.

WILSON William (1997) When Work disappears. The world of the new urban poor, New York, Alfred A. Knopf.

WONG David (1999) Geostatistics as measures of spatial segregation. Urban Geography, oㅡ 20, pp. 635-647.

WONG David (2003) Implementing spatial segregation measures in GIS, Computers, Environment and Urban Systems, nำ 27, pp. 53-70.

\section{NOTAS}

1. Utilizamos el concepto nueva inmigración para describir el fenómeno de la migración extracomunitaria que se emplea en las agriculturas mediterráneas (véanse Hoggart y Mendoza, 1999 ; Mottura, 1992; Pugliese, 1992). La nueva agricultura se ha convertido en uno de los sectores que actúan como polo de atracción de migrantes, hecho que la diferencia de las 
migraciones anteriores fordistas. La nueva migración se dirige a países, provincias o zonas que anteriormente habían sido expulsoras de mano de obra, caso de Almería. También, la incorporación de estos inmigrantes invierten la dirección habitual de los flujos desde el campo a la ciudad.

2. Hasta el momento nos hemos referido a viviendas o residencias, pero con la aparición de otros habitáculos residenciales que, en un principio, no tienen como objetivo albergar a la población utilizamos, también, el concepto de alojamiento.

3. Para el cálculo de las distancias nos hemos valido principalmente de fotos aéreas. En ningún caso hemos utilizado la línea recta, sino que hemos seguido los caminos o senderos que una persona debería recorrer para llegar desde un punto a otro. En otros casos hemos utilizado el cuentakilómetros del coche para medir las distancias.

4. Para la evaluación de las cualidades de la construcción contamos con la ayuda de un arquitecto que visitó con nosotros las viviendas de los inmigrantes encuestados.

5. El planteamiento de un entorno como dual, al igual que ocurre con el del mercado de trabajo, es reduccionista por sus lagunas empíricas y conceptuales. La ciudad se divide en más de dos segmentos. Nosotros utilizamos aquí esta acepción entendiéndola como los dos hábitats diferentes donde se insertan la población inmigrada y, no tanto, por las características y ubicación de las viviendas de los inmigrantes, frente a las de los autóctonos. Aunque para el caso almeriense, como veremos a lo largo del texto, la evidencia empírica nos muestra unos espacios muy dializados entre los españoles y los africanos.

6. El principal tipo de diseminado residencial toma el nombre de cortijo. Éstos fueron creados junto a las explotaciones agrícolas. El grueso de los cortijos situados en las zonas de agricultura intensiva de Almería se construyeron entre las décadas cincuenta y ochenta del siglo pasado periodo de despegue y consolidación de la agricultura en invernadero - y sus habitantes eran los propietarios de las explotaciones.

7.

25. -La concepción tradicional del problema de la vivienda ha sido definida básicamente como un desequilibrio entre lo necesitado y lo producido, sin tener en cuenta la relación o integración entre la vivienda y la estructura social. De ahí, que se proponga el concepto de residencia o de habitar (Cortés, 1995) como elemento aglutinador entre las dimensiones sociales y físicoespaciales.

26. Así, en ocasiones, en El Ejido o Roquetas de Mar con la intención de crear una "ciudad purificada", preocupaciones higienistas, moralizadoras o de insalubridad se convierten en causas para el desalojo de la casa, local o edificio, encubriendo el deseo de convertir esos hábitat en nuevos edificios, locales, etc. Y, por tanto, se enmascara un incipiente proceso de gentrification en nombre de una ciudad-jardín. En los diseminados por su lado, la cesión y ocupación actúan de bolsa de trabajadores en reserva y tanto los inmigrados como sus hábitats están "invisibles" y no necesitan tanto de políticas higienistas.

27. Lurbe (2004) se refiere a los inmigrados como poseedores de riesgo sanitario, donde el hacinamiento puede ser un factor importante.

28. A lo que hay que sumar que gran parte de los inmigrados mandan dinero a sus países de origen, reduciéndose más su capacidad de ahorro e inversión en materia de vivienda, que es suplida insertando nuevos individuos.

29. La población media de africanos por alojamiento es de 6,3 personas. También y según la Convención Europea de Colonia y el Comité Parker Morris del Reino Unido la superficie neta recomendada es de 98 metros cuadrados útiles. Sin embargo, la superficie media para nuestro caso es de 61 metros.

30. España es el país de la Unión Europea - de los quince - que menos gasta de su PIB en materia de vivienda (0,9\%), frente al 4,1 que gasta Suecia. Quedando el sector de la construcción y gestión inmobiliaria, exclusivamente, en manos de empresas privadas que no han puesto freno a la 
escalada de precios. Así, en España desde 1980 el precio de la vivienda se ha incrementado en un $726 \%$ en valor nominal y $124 \%$ en valor real (muy lejos del $33 \%$ real alemán), situación que ha obligado a los hogares a invertir casi todos sus ingresos en la vivienda.

31. En un $94 \%$ la cesión se produce en los diseminados que están junto a las explotaciones, el resto se da en algunas entidades locales dependientes, caso de Cortijos de Marín en Roquetas de Mar.

32. Evidentemente, cuando los inmigrados habitan un alojamiento en régimen de ocupación o cuando se realizan autoconstrucciones chabolísticas el pago en materia de alojamiento es nulo.

33. Otras investigaciones centradas también en el caso almeriense, utilizando diversas metodologías y procedimientos, describen la situación sobre la exclusión residencial, segregación y ocupación de infraviviendas por parte de los inmigrados como grave y extrema (véanse, entre otros, Martín y otros, 1999, Martínez, 1999, 2001 ; Foro Cívico, 2000 ; Defensor del Pueblo Andaluz, 2001 ; Girón, 2002 ; Checa, 2004) en cuanto a la exclusión residencial, segregación y la ocupación de infraviviendas de los inmigrados.

34. Para este caso hemos tomado como variable dependiente la habitabilidad.

\section{RESÚMENES}

En este artículo se describe la situación residencial de los inmigrantes procedentes de África en la provincia de Almería. Analizando dos aspectos, por un lado, la segregación residencial a partir de los índices de disimilaridad, aislamiento y absoluta centralización. Por otro, las características de los alojamientos habitados por los inmigrantes, tomando como referencia los indicadores de estabilidad, adecuación, habitabilidad y accesibilidad residencial. Los datos se obtienen del Padrón Municipal y la aplicación de una encuesta. Los resultados muestran una segregación residencial elevada y una situación residencial deficiente, donde casi tres de cada cuatro inmigrantes africanos ocupan una residencia no digna. El motivo principal que explica esta situación es la discriminación étnica.

Le voisin non désiré. Conditions de logement des immigrés africains à Almería (Espagne) Cet article décrit les conditions de logement des immigrés africains dans la province d'Almería. Nous aborderons d'une part la ségrégation résidentielle, mesurée par des indices de dissimilitude, d'isolement et de centralisation absolue. Nous présenterons ensuite les caractéristiques des logements habités par les immigrés, en nous référant aux indices de stabilité, adéquation, habitabilité et accès au logement. Les données proviennent du Recensement Municipal ainsi que de la réalisation d'une enquête. Les résultats témoignent d'une ségrégation résidentielle élevée ainsi que de conditions de logement insuffisantes (près de trois immigrés africains sur quatre vivent dans un logement indigne). Cette situation a comme facteur principal la discrimination ethnique.

The Not Accepted Neighbour. The African Immigrant's Residential Situation in Almería (Spain)

In this article the residential situation of the immigrants coming from Africa to the province of Almería is described, analyzing mainly two aspects. Firstly, the residential segregation starting from the indexes of dissimilarity, isolation and absolute centralization. Secondly, the characteristics of the lodgings inhabited by the immigrants, taking as reference the indicators of 
stability, adaptation, habitability and residential accessibility. The data are obtained from the Municipal Census and a survey. The results show a high residential segregation and a deficient residential situation, where almost three out of every four African immigrants live in a residence not deign. The main reason that explains this situation is the ethnic discrimination.

\section{ÍNDICE}

Mots-clés: Africains, conditions de vie, discrimination, logement, ségrégation Índice geográfico: Espagne

\section{AUTORES}

\section{JUAN CARLOS CHECA OLMOS}

Universidad de Almería, Departamento de Ciencias Humanas y Sociales, Carretera de Sacramento s/n. La Cañada de San Urbano -04120 Almería (España). jcheca@ual.es

\section{ÁNGELES ARJONA GARRIDO}

Universidad de Almería, Departamento de Ciencias Humanas y Sociales, Carretera de Sacramento s/n. La Cañada de San Urbano -04120 Almería (España). arjona@ual.es 